\title{
THE
}

\section{Choosing the Right Free IM Providers and Clients for Your Library}

Amanda Izenstark

University of Rhode Island, amanda@uri.edu

Follow this and additional works at: https://digitalcommons.uri.edu/lib_ps_pubs

Part of the Library and Information Science Commons

The University of Rhode Island Faculty have made this article openly available.

Please let us know how Open Access to this research benefits you.

This is a pre-publication author manuscript of the final, published article.

Terms of Use

This article is made available under the terms and conditions applicable towards Open Access

Policy Articles, as set forth in our Terms of Use.

Citation/Publisher Attribution

Izenstark, Amanda. "Choosing the Right Free IM Providers and Clients for Your Library." , (2009). doi: 10.1080/15228950802631127. 
Running head: Choosing the right free IM providers and clients for your library

Choosing the right free IM providers and clients for your library

\author{
Amanda K. Izenstark \\ Reference \& Instructional Design Librarian \\ University of Rhode Island
}




\begin{abstract}
This article describes the features and functionality of major instant messaging providers and clients as they relate to library needs, specifically for the provision of instant message reference services.
\end{abstract}

\title{
Choosing the right free IM providers and clients for your library
}

AIM or Yahoo! or MSN or Google? Trillian? Meebo? Pidgin? Adium? While the price is right -- free -- the world of instant messaging programs and tools can be baffling, and is, due to the popularity of open source programs for managing accounts, ever-changing. IM is a simple way to put access to library information right where patrons need it: online where they're researching. Libraries use IM to answer reference questions and to offer research guidance, but a number of libraries have separate accounts for their circulation desks and access services departments. Some libraries use IM to communicate internally as well. Selection of the most appropriate tools and services depends on a number of variables. Massive amounts of information regarding free IM providers and clients is available online, but for features libraries are more likely to want, some clients are more useful than others.

\section{Why Consider Free Tools?}


Whether budget has become an issue or this is a first step in providing instant messaging services for patrons, free clients offer flexibility and features that still allow provision of assistance at the point of need. Free clients are don't include some of the features that are attractive about virtual reference software, such as co-browsing, but unlike those programs, these do not require special software or separate logins on the user's end. Some of these services are operating system and browser independent, and your patrons probably already have one or more of these programs installed.

\section{Providers, Clients, and Aggregators}

A provider of IM service is the company with which the user has an account. Some of the most well-known providers are AOL Instant Messenger (AIM), Yahoo!, Microsoft's MSN, and Google Talk. Sometimes these names can be confused with the names of IM clients. Clients are the programs used to access instant messages, and the providers offer their own proprietary clients, many of which also serve to deliver ad content. AIM, Yahoo! Messenger, Windows Live Messenger, and Google Talk are the clients of the above, respectively. Sensing a need to manage multiple accounts on multiple clients, individuals and companies began creating clients that would do just that, and have added functionality. This has led to a number of possible configurations libraries can use to offer instant message reference, but through which providers? Which clients? What factors should drive the selection? 
First, what are patrons (and potential patrons) using? This question should drive any decision regarding instant messaging reference. AIM has a large market share, and is thus likely to be a provider patrons are already using. But it's not the only service available. Using an IM client program that monitors multiple services -- an aggregator allows easier and simultaneous management of accounts on multiple providers.

Consider the library's needs and goals. How is or will the service be staffed? If staffed by someone whose sole duty is to remain in front of the computer screen at all times anticipating incoming questions, perhaps an audio cue will not be necessary. If it's likely that coverage of the service is one of many tasks the person might be doing, then weigh the benefits of an audio cue and/or a visual cue, such as a flashing pop-up window.

Will staffers be monitoring the library's screen name at the same time that they are logged into their own? Some librarians offer their own work screen names to patrons or colleagues while monitoring incoming messages to a general screen name, so the ability to log in to the same service using multiple accounts (for example two different AIM accounts) is an important feature.

Some libraries would prefer to be able to use one account or screen name to reduce user confusion and have one point of access, but have multiple simultaneous logins.

Finally, how will the service be assessed? Do you need to save logs for later evaluation of services? How easy is it to locate transaction logs? 


\section{IM Providers}

Here is a quick review of some of the largest providers, and the features of interest to librarians. All of their client programs offer basic functionality including timestamps and message logging, and are available for Windows and Mac. Unfortunately, they don't generally allow two accounts on the same service to be logged in at the same time.

\section{AOL Instant Messenger (AIM)}

The instant messaging service from America OnLine allows multiple logins under the same screen name, making it easy for multiple librarians to monitor one screen name. When a second user logs in under the same screen name, both users receive a notification of simultaneous logins.

Yahoo! Messenger http://messenger.yahoo.com/

Unlike AIM, Yahoo! does not allow simultaneous logins. When a second user logs on to the same account, the first user may be disconnected without notice, so two staffers can not monitor the same account. Multiple accounts may be needed, which may be confusing to patrons.

\section{MSN Messenger, aka Windows Live Messenger}

MSN Messenger is associated with the Microsoft network, and is accessible through Hotmail accounts as well as through a program included with Windows XP and downloadable client software for Windows Vista and for the Mac. Does not allow 
simultaneous logins. Yahoo! and MSN allow users to add "buddies" from each others' services. For example, janedoe@yahoo.com can add johndoe@hotmail.com to her contacts list (and vice versa) without having a screen name on the other service.

Google Talk http://www.google.com/talk/

With its immense amount of storage space, simple interface, and brand recognition, Google's mail service, Gmail, rapidly gained popularity after its launch. It added a chat function for users, accessible through Gmail, on the web, or through a separate Windows-only client, and recently expanded the functionality to include a group chat function. Logs can be stored in the Gmail account, but if a user has chosen to go "Off the Record," their logs will not be recorded on either users' account.

\section{IM Client Aggregators}

All of the providers make it easy to download their own software to your client machines, but what if you want to monitor multiple services at once? Several clients allow you to do this, and most have the added benefit of being ad-free.

Trillian Basic (Windows only) Version 3.1.11.0 reviewed.

http://www.ceruleanstudios.com/ Trillian is a simple and popular aggregator that works with AIM, ICQ, Yahoo!, and MSN. Logs can be stored in the default location or in a custom folder, and are easy to access. When a user initiates a conversation, logs are stored in a text document under the user's screen name. If the user participates in subsequent transactions, additional logs are appended in chronological order, which 
making compilation of logs for analysis relatively simple. Trillian does have several drawbacks, however. First, user icons associated with screen names are stored in another folder and must be deleted manually. Second, sound settings are either all-on or all-off. This makes it impossible to have an audible notification when a new message comes in but not have audible confirmation during a session, a problem if staffing occurs at a desk in a public area. Trillian does allow users to manage multiple accounts on the same instant messaging service, such as a personal screen name and an institutional screen name.

Pidgin (Formerly GAIM; Linux/Unix or Windows platforms) Version 2.5.2 reviewed. http://www.pidgin.im Pidgin is very customizable. It is easy to set sound preferences so that alerts can sound for an initial incoming message from a patron, but not sound for subsequent messages while that window is open. Like Trillian, Pidgin allows users to manage multiple accounts on the same instant messaging service. Finally, Pidgin supports using AIM through SMS, which many libraries use to provide text reference services without needing a cell phone at the reference desk. Logs, however, can be difficult to locate. On Windows XP, for example, they are located in a hidden folder in the c:IDocuments and Settings|[user] directory and can't be found unless the folder's preferences are set to display hidden files and folders. Once there, the sub-folders are labeled with the screen name of each user who sends a message. Within these folders are text files labeled with just the date and time of the transaction. If compiling logs for analysis, the text files for multiple users can be moved to one folder, and then they can 
be sorted by the file names. Works with AIM, Google Talk, MSN, MySpace IM, Yahoo!, and more.

Adium (Mac Only) Version 1.3.2 reviewed. http://www.adiumx.com/ Has many of the same features as Pidgin, including customizable audio alerts and the ability to manage multiple screen names and multiple services. Like Pidgin, it can be difficult to locate the logs, but logs are sorted by user name and time of contact, making it relatively easy to sort logs. Unlike Pidgin, it does not support the AIM SMS service.

iChat (Mac Only) http://www.apple.com/macosx/features/ichat.html

iChat is the native instant messaging client for the Mac, but it supports only two providers: AIM and users with accounts on Apple's mac.com service. While the audio preferences are easily customizable and the logs are easy to access from the preferences menu, the sender's names are not consistently applied to the messages, making service evaluation difficult.

Digsby (Windows only) http://www.digsby.com

This aggregator goes beyond instant messaging to social networking sites. Digsby works with AIM, MSN, Yahoo!, Google Talk, ICQ, Jabber, and Facebook Chat. The interface is easy to use and logs are very easy to access through a "Past Chat Browser" from the Tools menu. Digsby also has a widget that can be embedded on web pages for easy contact. Unfortunately, the program uses a significant amount of memory when 
running, which may slow down older computers. Like Trillian, Digsby does not support the AIM hack.

Meebo (Web-based, with optional Firefox extension) http://www.meebo.com/ Meebo is primarily an ad-supported web-based service that allows users to log in to AIM, Yahoo!, MSN, Google Talk and more all at once, without the need to install software. Because Meebo is web-based, it is a simple solution for libraries where instant messaging services have been blocked. One of Meebo's popular features is the MeeboMe widget, which can be used to put access to a librarian at the point of need: on a web page where users may be searching. Users do not need to download software, nor do they have to have a screen name or register in order to contact someone using a MeeboMe widget. Meebo has some drawbacks that limit its usability for libraries, however. The MeeboMe widget does not include a logging function, so it's impossible to obtain statistics or logs of contact with guest users through the widget for analysis. Because contacting a user via the widget does not require a user name, some libraries have reported unwanted or harassing messages via the chat widget. Although Meebo does not have a standalone client (an unauthorized client has been created on the Adobe Air platform), Meebo has released a Firefox extension that simplifies logging in to Meebo, but still requires that a Meebo tab remain open, and consumes some space in the browser window.

\section{On the horizon:}


Libraryh3lp is a free client under development that includes support for the major instant messaging services, with additional features geared toward library use including queuing and routing of questions to specific librarians. The developers plan to charge a small fee for the final product. (Sessoms, 2008) Trillian Astra's web interface promises to include both IM and social networking sites, making it easier for libraries to monitor their Facebook and MySpace accounts, but is currently in closed beta. Finally, as of March 2008, the developers of Digsby noted that Mac and Linux versions would be released in approximately a year.

\section{Logging and Security}

Many libraries consider logging an essential feature to garner usage statistics, ensure service quality, and use in training, but it is wise to consider not keeping logs, and to purge logs that are kept frequently. If an IM client automatically adds patrons to the "contacts" or "buddy list," make it a habit to remove users on a regular basis. Patrons will not be notified that they are removed, but take care not to accidentally "block" a patron, or s/he will be unable to send messages to the service.

In short, weigh institutional needs as well as those of patrons when considering what programs to implement. In some instances, IT staff will have had experience with various tools and may have recommendations, although some institutions block some IM programs due to virus concerns. With the price being free, and with IM being a lowthreshold technology, nearly any library can experiment with instant messaging. 


\section{References}

Sessoms, P. (2008, April 29). Free_or_fee_full_disclosure: What's the deal? Is this going to be free forever? Google Code: Libraryh3/p. Retrieved November 8, 2008 from http://code.google.com/p/libraryh3lp/wiki/Free_or_Fee_Full_Disclosure 